\title{
Study on loss mechanism of SMA tracheal stent subjected to cough excitation
}

\author{
Zhiwen Zhu ${ }^{\mathrm{a}}$, Xinmiao $\mathrm{Li}^{\mathrm{a}}$ and Jia $\mathrm{Xu}^{\mathrm{a}, \mathrm{b}, *}$ \\ ${ }^{a}$ Deparment of Mechanics, Tianjin University, 92 Weijin Road, Nankai District, Tianjin 300072, \\ China \\ ${ }^{b}$ Tianjin Key Laboratory of Nonlinear Dynamics and Chaos Control, 92 Weijin Road, Nankai District, \\ Tianjin 300072, China
}

\begin{abstract}
A kind of Ti-Ni shape memory alloy (SMA) hysteretic nonlinear model is developed, and the loss mechanism of a SMA tracheal stent subjected to cough excitation is studied in this paper. Nonlinear differential items are introduced to express the hysteretic phenomena of Ti-Ni SMA, and the fitting effect of the SMA constitutive model on the experimental data is proved by the partial least-square regression method. The nonlinear dynamic model of a Ti-Ni SMA tracheal stent subjected to cough excitation is developed, and the system's dynamic response is obtained. The numerical results show that the system's vibration is little in weak excitation, becomes large with the increase of the stochastic excitation, and finally becomes little again with the further increase of the stochastic excitation; the stochastic resonance phenomenon occurs in the process, which may cause stent fracture or loss.
\end{abstract}

Keywords: Shape memory alloy (SMA), tracheal stent, cough, stochastic resonance

\section{Introduction}

Tracheal stenosis is a kind of complication induced by the benign or malignant lesions in and around the trachea. It has poor prognosis and high mortality. Airway stenting has been the most commonly used method for the relief of tracheal stenosis. Shape Memory Alloy (SMA) is an ideal biomedical engineering material due to its excellent corrosion resistance and biocompatibility. Ti-Ni SMA tracheal stents have been applied widely to treat tracheal stenosis [1]. Stent loss becomes a serious problem with the increase of tracheal stents' application. When a tracheal stent is implanted into a trachea, it is fixed by tension and friction force [2]. When a patient coughs roughly, the contraction and relaxation of his tracheal smooth muscles become violent, which exert the great radial pressure to the tracheal stent. It means that the nonlinear deformation of the tracheal stent must be considered. Moreover, the cough process is not harmonic, and there is random fluctuation in the cough's intensity. Thus, the contract and relax force belongs to the bounded noise excitation, which is combined by harmonic force and stochastic force. Subjected to the great radial bounded noise excitation, SMA tracheal stents have complex response, which causes its vibration and loss. To avoid

\footnotetext{
* Address for correspondence: Jia Xu, Department of Mechanics, Tianjin University, 92 Weijin Road, Nankai District, Tianjin 300072, China. Tel.: +8627401981; Fax:+8627401981; E-mail: xujia@tju.edu.cn.
} 
the stent loss, it is necessary to study the nonlinear dynamic characteristics of a SMA tracheal stent subjected to radial bounded noise excitation.

Many researchers have studied the applications of tracheal stents in biomedical engineering [3-10]. Montgomery proposed T-tube tracheal stent firstly [3]. George, et al. applied a covered expandable metal stent for recurrent tracheal obstruction [4]. Carrasco et al. managed the tracheal and bronchial stenoses with the Gianturco stent [5]. Vergnon, et al. assessed the efficacy of tracheal and bronchial stent placement on respiratory functional tests [6]. Yanagihara et al. treated the tracheal stenosis with self-expanding nitinol stent [7]. Bolliger, et al. applied a new self-expandable silicone stent in an experimental tracheal stenosis [8]. Vergnon, et al. evaluated preliminarily the efficacy and tolerance of a new silicone stent for the treatment of benign tracheal stenosis [9]. Mittleman, et al. studied the fracture of an endoluminal nitinol stent used in the treatment of tracheal collapse in a dog [10]. Although many achievements have been reported in the past decades, most of them were focused on design and efficacy evaluation, while the theoretical results of the dynamic characteristics of tracheal stent were limited. In this paper, the nonlinear dynamic characteristics of a Ti-Ni SMA tracheal stent subjected to cough excitations are studied, and its loss mechanism is analyzed.

For obtaining the dynamic characteristics of a Ti-Ni SMA tracheal stent in cough process, it is necessary to establish an accurate constitutive model of Ti-Ni SMA. Most of the current SMA constitutive models are presented as equations with piecewise functions because of the hysteretic characteristics of Ti-Ni SMA. As results, it is hard to analyze the dynamic characteristics of the SMA tracheal stent in theory. In this paper, nonlinear differential items are introduced to explain the hysteretic loop of Ti-Ni SMA strain-stress curves, and the dynamic characteristics of a Ti-Ni SMA tracheal stent subjected to radial bounded noise excitation are analyzed.

\section{Material and methods}

The strain-stress curves of Ti-Ni SMA are presented in Figure 1. In this paper, a new nonlinear differential model is introduced to explain the hysteretic loop of Ti-Ni SMA as follows:

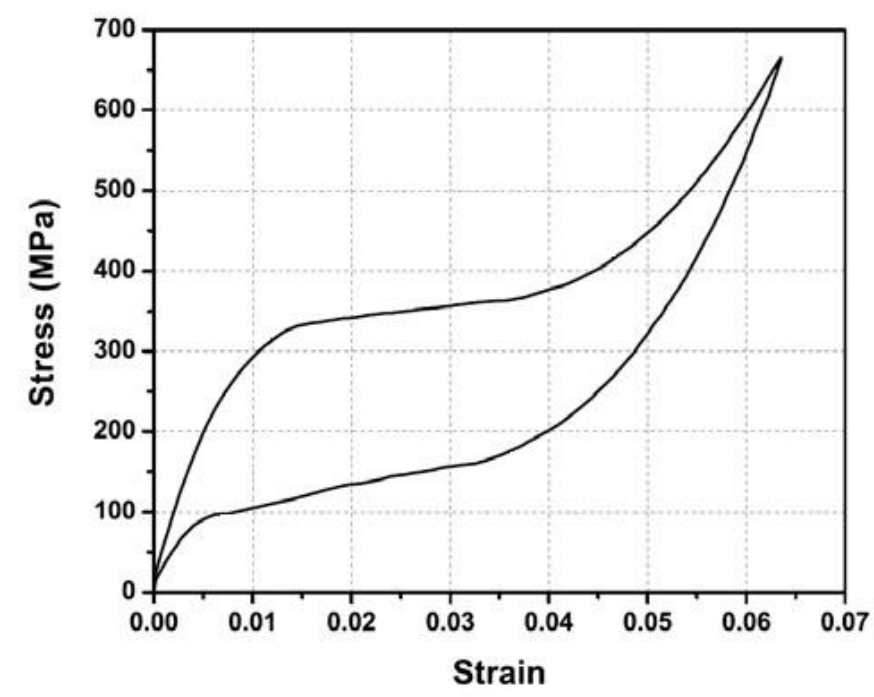

Fig. 1. Strain-stress curves of Ti-Ni SMA. 


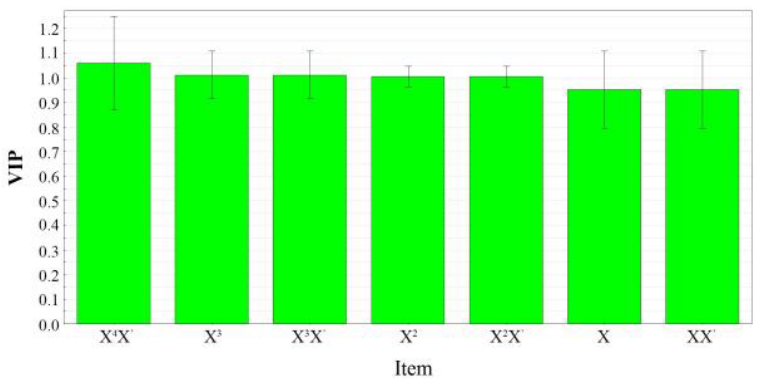

Fig. 2. Variable importance of each item.

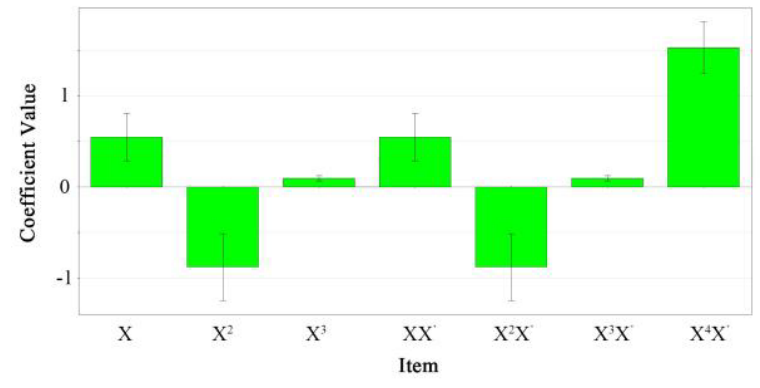

Fig. 3. Coefficient values of each item.

$$
\sigma=\sigma_{1}+\sigma_{2}=a_{1} \varepsilon+a_{2} \varepsilon^{2}+a_{3} \varepsilon^{3}+\left(a_{4} \varepsilon+a_{5} \varepsilon^{2}+a_{6} \varepsilon^{3}+a_{7} \varepsilon^{4}\right) \dot{\varepsilon}
$$

where $\sigma$ is the stress, $\varepsilon$ is the strain, $a_{i}(\mathrm{i}=1 \sim 7)$ are the coefficients, $\sigma_{1}=a_{1} \varepsilon+a_{2} \varepsilon^{2}+a_{3} \varepsilon^{3}$ is the skeleton curve of the hysteretic loop, $\sigma_{2}=\left(a_{4} \varepsilon+a_{5} \varepsilon^{2}+a_{6} \varepsilon^{3}+a_{7} \varepsilon^{4}\right) \dot{\varepsilon}$ is the new nonlinear differential item, which is developed from $[11,12]$.

The partial least-square regression (PLSR) method is used to verify the forecasting results of Eq. (1). The PLSR method is a kind of numerical statistics method, which is usually applied in fitting test. The analysis results based on the experimental data are presented in Figure 2, and the coefficient values of each item are presented in Figure 3, where VIP is the variable importance. There is no item whose VIP is evidently little, which means that all items should be reserved in the constitutive model.

The results of fitting test to Eq. (1) are presented in Figure 4, in which the red lines present the experimental data, and the black lines present the forecast values. Eq. (1) can explain the experimental curves well.

The mechanical model of a Ti-Ni SMA tracheal stent subjected to radial bounded noise excitation is shown in Figure 5. The structure can be simplified as thin cylindrical shell. The dynamic model of a Ti-Ni SMA tracheal stent subjected to radial bounded noise excitation can be shown as follows:

$$
\rho h r^{2} \frac{\partial^{2} w}{\partial t^{2}}=\frac{\partial}{\partial \theta}\left[N_{\theta} \frac{\partial w}{\partial \theta}+\lambda N_{x \theta} \frac{\partial w}{\partial \xi}+Q_{\theta z}\right]-N_{\theta}+\frac{M_{\theta}}{r}+q r
$$

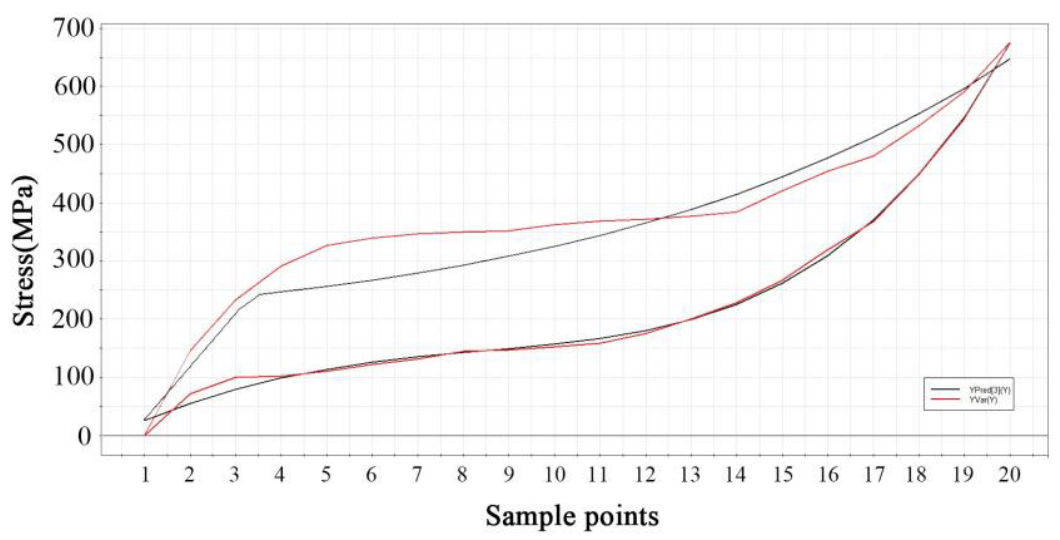

Fig. 4. Results of fitting test for Eq. (1) on strain-stress data of SMA. 


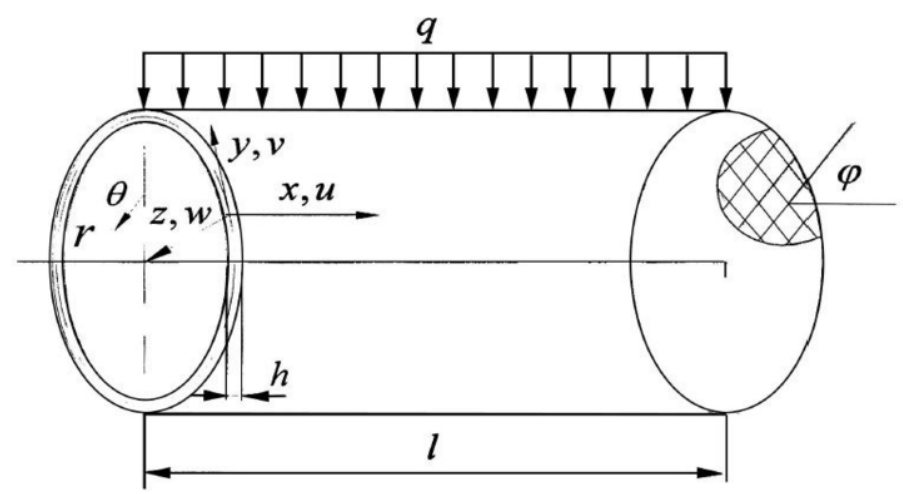

Fig. 5. Mechanical model of Ti-Ni SMA tracheal stent.

where $\rho$ is the density, $h$ is the thickness, $r$ is the radius, $w$ is the vibration mode, $w=w(t, \theta)=u(t) \sin k \theta, \theta$ is the torsion angle, $N_{\theta}$ is the shearing stress, $\lambda=r / l$ is the aspect radio, $\xi=x / l, M_{\theta}$ is the torque, $q=q(t)=F \sin (\Omega t+\chi+\sigma B(t))$ is the bounded noise, $F$ is the intensity of radial pressure, $B(t)$ is Gauss white noise, $\chi$ is the uniformly distributed random phase between $[0,2 \pi), \sigma$ is the intensity of $B(t)$. Thus, the dynamic equation of radial displacement can be obtained from Eq. (2) by Galerkin's method as follows:

$$
\ddot{u}+\left(2 \eta+b_{3} u^{2}+b_{4} u^{4}\right) \dot{u}+b_{1} u+b_{2} u^{3}=e u \sin (\Omega t+\chi+\sigma B(t))
$$

where $b_{1}=\frac{a_{1} I_{1} \pi^{4}}{\rho h r^{2}}, b_{2}=\frac{3 a_{3} I_{3} \pi^{8}}{4 \rho h r^{2}}, \eta=\frac{c}{2 \rho h r^{2}}, b_{3}=\frac{3 a_{5} I_{3} \pi^{8}}{4 \rho h r^{2}}, b_{4}=\frac{5 a_{7} I_{5} \pi^{12}}{8 \rho h r^{2}}, e=\frac{F \pi^{2}}{\rho h r^{2}}$.

\section{Nonlinear dynamic characteristics of a Ti-Ni SMA tracheal stent subjected to bounded noise}

The averaged Ito equation of Eq. (3) can be obtained by the stochastic average method as follows:

$$
\left\{\begin{array}{l}
d A=m_{1}\left(A, \Delta^{\prime}\right) d t \\
d \Delta^{\prime}=m_{2}\left(A, \Delta^{\prime}\right) d t+\sigma d B(t)
\end{array}\right.
$$

where $A, \Delta^{\prime}$ are both the two-dimensional diffusion processes,

$$
\begin{gathered}
\Delta^{\prime}=\Omega t+\sigma B(t)+\chi-\Theta \\
m_{1}\left(A, \Delta^{\prime}\right)=-\pi\left(b_{3} A^{2}+\frac{1}{4} b_{4} A^{4}\right)-\frac{e A}{2 \eta} \cos \Delta^{\prime} \\
m_{2}\left(A, \Delta^{\prime}\right)=2 \pi \Omega-\frac{1}{\eta}\left(\frac{3}{4} \pi b_{2} A^{3}-\frac{e}{2} \sin \Delta^{\prime}\right)
\end{gathered}
$$




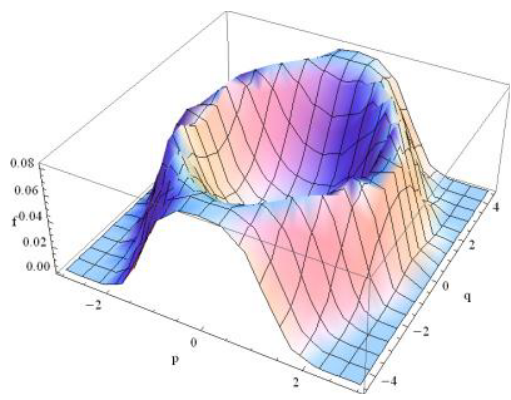

(a) $e=0.1$

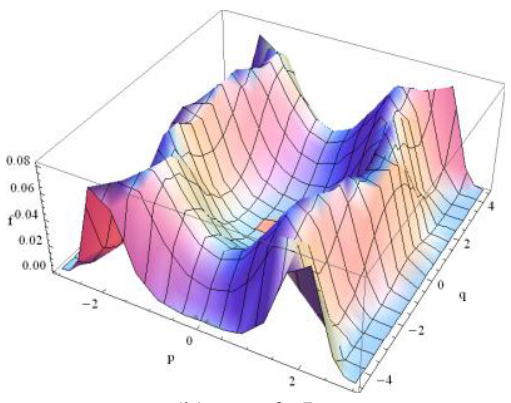

(b) $e=0.5$

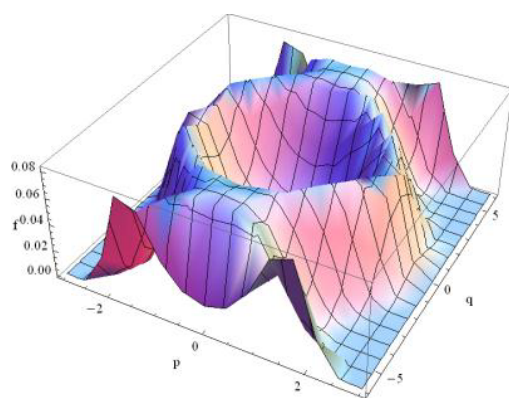

(c) $e=0.8$

Fig. 6. Stationary probability density of the system's response.

The averaged Fokker-Planck-Kolmogolov (FPK) equation on the stationary probability density (SPD) of the system's response $f=f\left(A, \Delta^{\prime}, t\right)$ can be presented as follows:

$$
\frac{\partial f}{\partial t}=-\frac{\partial}{\partial A}\left(m_{1} f\right)-\frac{\partial}{\partial \Delta^{\prime}}\left(m_{2} f\right)+\frac{\sigma^{2}}{2} \frac{\partial^{2} f}{\partial \Delta^{2}}
$$

The stationary probability density of the system's response are presented in Figure 6, where $\eta=0.08, b_{1}=1, b_{2}=0.2, b_{3}=0.1$, and $b_{4}=0.008$. As the figures show, the noise intensity $e$ has important influence on the system's dynamical response. When the noise is weak, it has little effect on the system's response; the system becomes accidentally unstable with the increasing of the noise's intensity; when the noise's intensity further increases, the system's response becomes stable again as before.

The numerical results of a Ti-Ni SMA tracheal stent subjected to radial bounded noise excitation are shown in Figures 7-9, where the cough frequency $\Omega=3 \mathrm{~Hz}$. According to the experimental results, we can see that the mean vibration amplitude is 3.1 when $e=0.1$, rises to 4.07 when $e=0.5$, and finally is 3.5 when $e=0.8$. It means that the system's response is little in weak excitation, and then increases with the excitation, finally decreases with the further increase of the excitation. The system we studied in this paper is a stochastic dynamic system. Thus, stent loss will occur in probability when stochastic resonance phenomenon happens. From Figure 8, we can see that the stochastic resonance phenomenon occurs when $e=0.5$. It means that the vibration amplitude of SMA stent will be significant in this case, which cause the stent fracture or loss. The results of simulation are accord with the theoretical analysis.

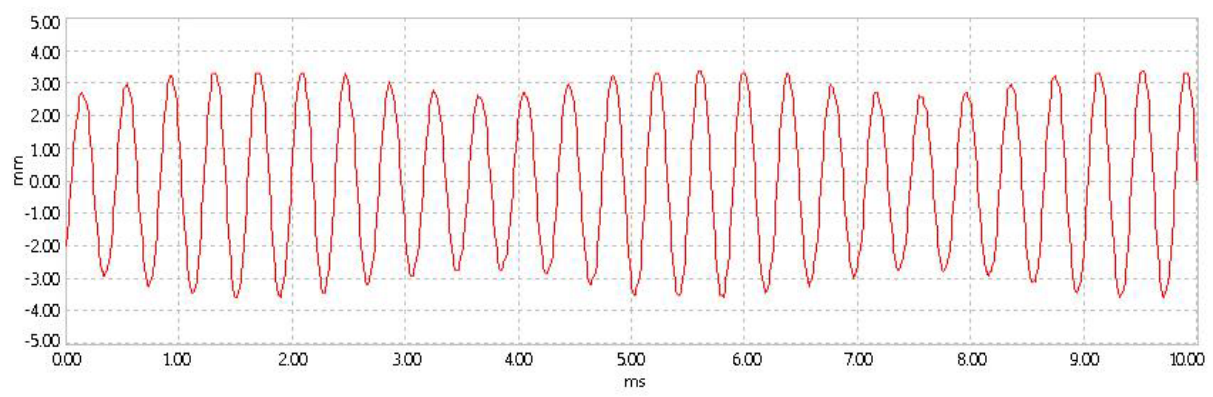

Fig. 7. Response of Ti-Ni SMA tracheal stent when $e=0.1$. 


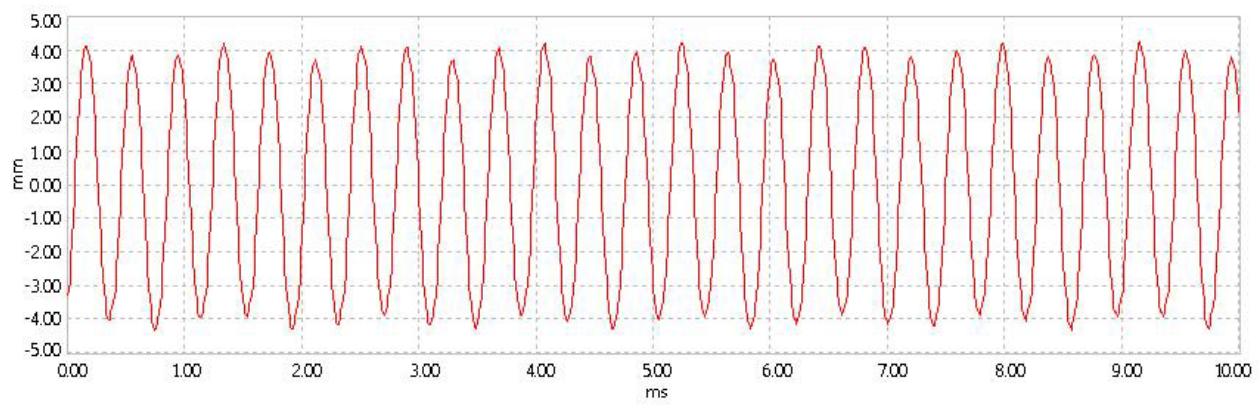

Fig. 8. Response of Ti-Ni SMA tracheal stent when $e=0.5$.

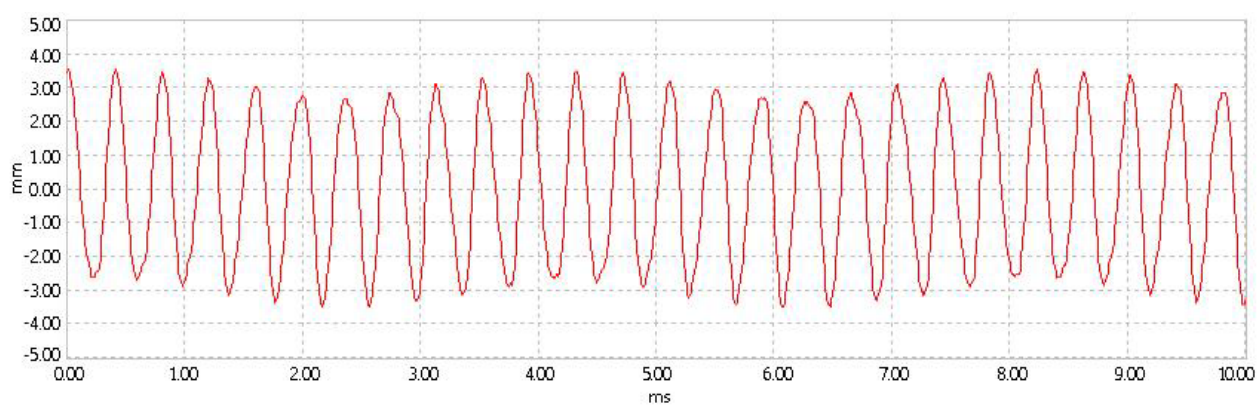

Fig. 9. Response of Ti-Ni SMA tracheal stent when $e=0.8$.

\section{Conclusion}

A kind of Ti-Ni SMA's hysteretic nonlinear model is developed, and the loss mechanism of a Ti-Ni SMA tracheal stent subjected to cough excitation is studied in this paper. Nonlinear differential items are introduced to explain the Ti-Ni SMA's hysteretic phenomena, and the fitting effect of the constitutive model on the experimental data is proved by the partial least-square regression method. The nonlinear dynamic model of a SMA tracheal stent subjected to cough excitation is developed, and the system's dynamic response is obtained. The numerical results show that the system's response is little in weak excitation, then becomes large with the increase of the stochastic excitation, and finally becomes little again with the further increase of the stochastic excitation; the stochastic resonance phenomenon occurs in the process, which may cause stent fracture or loss.

\section{Acknowledgment}

The authors gratefully acknowledge the support of the Natural Science Foundation of China (NSFC) through Grant Nos. 11272229 and 11302144, the Ph.D. Programs Foundation of the Ministry of Education of China through Grant No. 20120032120006, and the Tianjin Research Program of Application Foundation and Advanced Technology through Grant No. 13JCYBJC17900, 14JCQNJC05300, and 14JCQNJC05600. 


\section{References}

[1] T. Nakamura, Y. Shimizu and T. Matsui, Novel airway stent using a thermal shape-memory Ti-Ni alloy, American Society for Artificial Internal Organs Transactions 3 (1991), 319-321.

[2] V. Siddharth, E. Amanda and C. Timothy, Determination of coefficient of friction for self-expanding stent-grafts, Journal of Biomechanical Engineering-Transactions of American Society of Mechanical Engineers 12 (2010), 1210076.

[3] W.W. Montgomery, T-tube tracheal stent, Archives of Otolaryngology 3 (1965), 320-321.

[4] P.J. George, J.D. Irving and B.S. Mantell, Covered expandable metal stent for recurrent tracheal obstruction, Lancet 8689 (1990), 582-584.

[5] C.H. Carrasco, J.C. Nesbitt and C. Charnsangavej, Management of tracheal and bronchial stenoses with the Gianturco stent, The Annals of Thoracic Surgery 4 (1994), 1012-1017.

[6] J.M. Vergnon, F. Costes and M.C. Bayon, Efficacy of tracheal and bronchial stent placement on respiratory functional tests, Chest 3 (1995), 741-746.

[7] K. Yanagihara, H. Mizuno and H. Wada, Tracheal stenosis treated with self-expanding nitinol stent, The Annals of Thoracic Surgery 6 (1997), 1786-1789.

[8] C.T. Bolliger, C. Wyser and X.R. Wu, Evaluation of a new self-expandable silicone stent in an experimental tracheal stenosis, Chest 2 (1999), 496-501.

[9] J.M. Vergnon, F. Costes and J.C. Polio, Efficacy and tolerance of a new silicone stent for the treatment of benign tracheal stenosis-Preliminary results, Chest 2 (2000), 422-426.

[10] E. Mittleman, C. Weisse and S.J. Mehler, Fracture of an endoluminal nitinol stent used in the treatment of tracheal collapse in a dog, Journal of the American Veterinary Medical Association 8 (2004), 1217-1221.

[11] Z.W. Zhu, Q.X. Zhang and J. Xu, Nonlinear dynamic characteristics and optimal control of giant magnetostrictive filmshape memory alloy composite plate subjected to in-plane stochastic excitation, Chinese Physics B 8 (2014), 088201.

[12] Z.W. Zhu, Q.X. Zhang and J. Xu, Nonlinear dynamic characteristics of SMA intravascular stent under radial stochastic loads, Bio-Medical Materials and Engineering 1 (2014), 483-489. 